\title{
La problématique de la lecture documentaire du texte informationnel
}

De la connaissance communiquée à la connaissance partagée

The Problems Associated With the Reading of a Text

From Communicated Knowledge to Shared Knowledge

\section{La problemática de la lectura documentaria del texto}

"informational"

\section{Del conocimiento comunicativo al conocimiento compartido}

\author{
Hubert Fondin
}

Volume 52, numéro 1, janvier-mars 2006

URI : https://id.erudit.org/iderudit/1030024ar

DOI : https://doi.org/10.7202/1030024ar

Aller au sommaire du numéro

\section{Éditeur(s)}

Association pour l'avancement des sciences et des techniques de la documentation (ASTED)

\section{ISSN}

0315-2340 (imprimé)

2291-8949 (numérique)

\section{Découvrir la revue}

\section{Citer cet article}

Fondin, H. (2006). La problématique de la lecture documentaire du texte informationnel : de la connaissance communiquée à la connaissance partagée. Documentation et bibliothèques, 52(1), 17-28. https://doi.org/10.7202/1030024ar
Résumé de l'article

La lecture informative d'un texte est une lecture de questionnement pour trouver réponse à un besoin informationnel. Réalisée pour quelqu'un d'autre, dans un cadre professionnel, c'est une lecture documentaire.

La lecture informative d'un texte est la lecture que chacun fait. Est-elle donc par essence "subjective » ? Beaucoup acceptent cela quand elle est réalisée pour soi. C'est celle du lecteur final. Mais plusieurs le refusent catégoriquement quand elle est réalisée pour autrui. C'est celle du lecteur intermédiaire, celle qui est faite dans un cadre professionnel comme la documentation. Ils considèrent alors que la lecture documentaire doit être " objective ». Dès lors ils établissent implicitement ou explicitement que le lecteur n'a aucune liberté de choix dans sa lecture.

Nous allons d'abord considérer la lecture en tant qu'activité de recherche de sens, en visitant successivement les sens possibles, le sens voulu par l'auteur, le sens inscrit dans le texte et le sens attendu par le lecteur. Puis nous exposerons comment, sur le plan théorique, une vision " vectorielle » de la communication, en référence au schéma de C. E. Shannon, explique le souci d'objectivité des documentalistes, puisque l'information est la connaissance à communiquer. Enfin nous montrerons comment une vision « interactive ", issue de la contestation de ce schéma " classique », permet aux chercheurs en science de l'information de considérer différemment la lecture " documentaire », car l'information, à travers cette lecture, devient une connaissance partagée.
Tous droits réservés (c) Association pour l'avancement des sciences et des techniques de la documentation (ASTED), 2006
Ce document est protégé par la loi sur le droit d'auteur. L'utilisation des services d'Érudit (y compris la reproduction) est assujettie à sa politique d'utilisation que vous pouvez consulter en ligne.

https://apropos.erudit.org/fr/usagers/politique-dutilisation/ 


\title{
De la connaissance communiquée à la connaissance partagée
}

\author{
HUBERT FONDIN \\ Université de Bordeaux 3 \\ Hubert.Fondin@u-bordeaux3.fr
}

\section{RÉSUMÉ | ABSTRACTS | RESUMEN}

La lecture informative d'un texte est une lecture de questionnement pour trouver réponse à un besoin informationnel. Réalisée pour quelqu'un d'autre, dans un cadre professionnel, c'est une lecture documentaire.

La lecture informative d'un texte est la lecture que chacun fait. Est-elle donc par essence "subjective»? Beaucoup acceptent cela quand elle est réalisée pour soi. C'est celle du lecteur final. Mais plusieurs le refusent catégoriquement quand elle est réalisée pour autrui. C'est celle du lecteur intermédiaire, celle qui est faite dans un cadre professionnel comme la documentation. Ils considèrent alors que la lecture documentaire doit être "objective». Dès lors ils établissent implicitement ou explicitement que le lecteur n'a aucune liberté de choix dans sa lecture.

Nous allons d'abord considérer la lecture en tant qu'activité de recherche de sens, en visitant successivement les sens possibles, le sens voulu par l'auteur, le sens inscrit dans le texte et le sens attendu par le lecteur. Puis nous exposerons comment, sur le plan théorique, une vision "vectorielle» de la communication, en référence au schéma de C. E. Shannon, explique le souci d'objectivité des documentalistes, puisque l'information est la connaissance à communiquer. Enfin nous montrerons comment une vision "interactive», issue de la contestation de ce schéma "classique», permet aux chercheurs en science de l'information de considérer différemment la lecture "documentaire», car l'information, à travers cette lecture, devient une connaissance partagée.

\section{The Problems Associated With the Reading of a Text: From} Communicated Knowledge to Shared Knowledge

The informational reading of a text is the type of reading that asks questions in order to satisfy an information need. Carried out for someone else, in a professional context, it becomes a documented reading.

The informational reading of a text can be carried out by everyone. Is it then necessarily "subjective"? Many would believe so when it is done for oneself; done by a single reader. But others refuse this assumption when its carried out for someone else by an intermediary reader in a professional context such as documentation. It is then considered "objective". From that point, it is implicitly or explicitly established that the reader has no freedom of choice or interpretation when he reads the text.
We will begin by considering reading as an activity that seeks meaning - the intended meanings, the meanings of the author, the meaning written in the text and the intended meaning of the reader. Then we will develop, on a theoretical level, a vectorial vision of communication based on C. E. Shannon, explaining the concerns for objectivity by documentalists, given that information is knowledge to be communicated. Finally, we will demonstrate how an interactive vision, emerging in opposition to the classic vision, allows researchers in information science to consider document reading in a different light because information, through this type of reading, becomes shared knowledge.

\section{La problemática de la lectura documentaria del texto "informacional".}

Del conocimiento comunicativo al conocimiento compartido

La lectura informativa de un texto es una lectura crítica que busca respuestas a una necesidad de información. Esta misma lectura realizada por otra persona en un marco profesional es más bien una lectura documentaria.

La lectura informativa de un texto es la lectura que cada uno realiza. Entonces, ¿es subjetiva por esencia? Muchos opinan que sí cuando la lectura es para ellos mismos: la lectura del lector final. Pero, opinan categóricamente lo contrario cuando otra persona la realiza: la lectura del lector intermediario, la que se realiza en un marco profesional como parte de la documentación. Se considera que la lectura documentaria debe ser objetiva, estableciéndose implícita o explícitamente que el lector no tiene ninguna libertad de elección en su lectura.

En este estudio, se considera primero la lectura como actividad de investigación del sentido, con sus posibles sentidos: el deseado por el autor, el inscrito en el texto y el esperado por el lector. Luego, se expone cómo una visión "vectorial" de la comunicación en referencia al esquema de C.E. Shannon, en el plano teórico, puede explicar la preocupación de la objetividad de los documentalistas puesto que la información es el conocimiento comunicativo. Finalmente, se muestra cómo una visión "interactive", resultante de la contestación de este esquema clásico, permite a los investigadores en ciencia de la información considerar de diferente manera la lectura "documentaria", puesto que la información a través de esta lectura se convierte en un conocimiento compartido.

pertinents. Longtemps son processus a été négligé, car elle est considérée comme naturelle, évidente: savoir lire un texte, c'est le comprendre. Il est vrai qu'en tant qu'activité fondamentalement intellectuelle et personnelle, elle est difficile à expliquer et donc à transmettre. Selon Philippe Sollers, c'est l'acte le plus individuel qui soit $^{2}$.

\footnotetext{
2. La lecture et sa voix - Le Monde des livres, 21 juillet 1995, p.11. Sans doute, parle

$\mathrm{t}$-il de la lecture-plaisir.
}

1. Même si parfois nous ne parlons que de "texte», il s'agit toujours d'un texte à contenu informationnel, comme les articles scientifiques. De même, lorsque nous parlons de lecture, elle est toujours de type «documentaire». 


\section{Tableau 1. Lecture et attribution de sens}

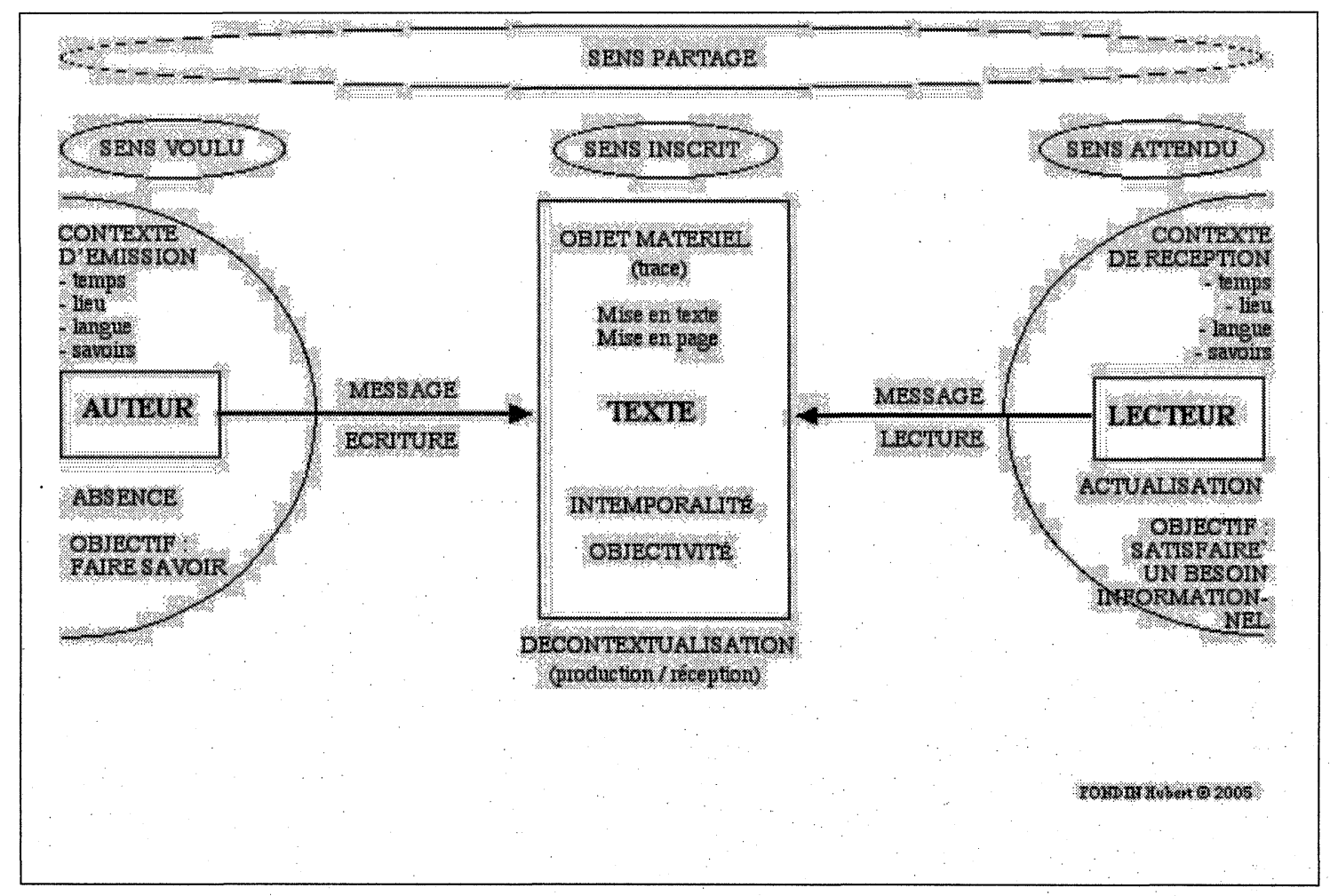

Pour autant, la lecture informative d'un texte est-elle la lecture que chacun fait. Est-elle donc par essence "subjective»? Beaucoup acceptent cela quand elle est réalisée pour soi. C'est celle du lecteur final. Mais le refusent catégoriquement quand elle est réalisée pour autrui. C'est celle du lecteur intermédiaire, celle qui est faite dans un cadre professionnel comme la documentation. Ils considèrent alors que la lecture documentaire doit être «objective». Dès lors ils établissent implicitement ou explicitement qu'il n'a aucune liberté de choix dans sa lecture.

Les choses sont-elles aussi simples dès lors que l'être humain, documentaliste ou autre, est sollicité comme intermédiaire? Peut-on admettre le caractère intrinsèque d'«objectivité » pour toute lecture d'un texte informationnel. C'est ce que semblent laisser entendre les documentalistes qui, par "principe», ne voient souvent même pas l'intérêt de ce questionnement. Du fait de leur rôle, ils doivent extraire le contenu «réel» du texte, naturellement le même pour tous. Ils lisent justement, donc objectivement. Ils choisissent sûrement les clés de recherche, et produisent les résumés utiles. Mais n'est-ce pas là une illusion de croire que la lecture, et la réécriture qui s'ensuit ${ }^{3}$, grâce aux définitions, grâce aux normes, grâce aux outils (thésaurus), grâce au savoir-faire, sont indiscutables? Une illusion qui justifie, pour d'autres, le recours à des machines qui établiront automatiquement les résumés et extrairont les mots-clés d'un texte? Or cette illusion nous parait directement associée à un cadre de référence utilisé implicitement, celui qui fonde le «principe». Ce cadre induit une conception de la lecture documentaire et, ce faisant, une pratique qui est, selon nous, objet à discuter.

\section{La recherche de sens ${ }^{4}$}

Lire est une activité stratégique de compréhension, donc de recherche de sens. Lire c'est faire du sens, c'est rendre intelligible, c'est intégrer, c'est comprendre ce qu'on lit. C'est, selon E. D. Hirsch, une démarche pour trouver ce qui reste stable dans la réception d'un texte. C'est la réponse à: "Que veut dire ce texte?» C'est, pour Jean Cervoni ${ }^{6}$, une «interprétation globale d'une production langagière, d'un énoncé». C'est la visée et le résultat de l'interprétation. Il faut insister sur le caractère «global», sur le fait que la représentation à construire doit englober la totalité du message.
3. Contrairement à la profession qui utilise l'expression «analyse documentaire», nous préférons parler de «lecture» et de «réécriture» documentaires, opposant de la sorte à une vision analytique, une approche interactive entre deux processus interdépendants, mais dissociables. C'est une illustration de la problématique de cet article.
4. Sur ce point, la lecture du livre d'Antoine COMPAGNON: Le Démon de la théorie. Littérature et sens commun - Paris: Seuil-Points, 1998 - 338 p., a été, pour nous, tout à fait stimulante.

5. The Aims of interpretation - Chicago: Univ. Chicago Press - 1976.

6. CERVONI, Jean - L'Énonciation - Paris: PUF, 1987 - p. 17-18. 
Tout lecteur, intermédiaire ou final, face à un texte dont il doit rechercher le sens, peut normalement se demander: "Que veut dire l'auteur?» "Qu'y a t-il dans ce texte?» «Qu'est-ce que je comprends?» Trois questions apparemment équivalentes. Mais le sontelles vraiment? Le sens d'un texte peut en effet être soit celui qui reflète l'intention de son auteur - sens voulu par celui qui l'a écrit; soit celui qui est contenu dans ce texte - sens inscrit et conservé sur un quelconque support; soit, enfin, celui qui est dégagé par le lecteur - sens perçu par celui qui le lit. Dès lors, quel est le poids de l'intention de l'auteur? Quelle est la place du texte? Quelle est la liberté du lecteur? On retrouve les trois éléments-clés du schéma de C. E. Shannon: l'émetteur, le message et le récepteur, chacun pouvant être regardé indépendamment des autres. En privilégier un, n'est peut-être pas si innocent que cela.

\subsection{Le sens d'un texte est celui voulu par l'auteur}

"Le sens d'un texte, c'est ce que l'auteur de ce texte a voulu dire ${ }^{7}$.» C'est le «vouloir-dire». La recherche de «sens» concerne le «sujet» du texte et la façon dont il est traité, c'est-à-dire tout le contenu intentionnel, le discours, voulu par l'auteur pour son texte dans une situation précise.

La lecture doit alors être aussi proche que possible de celle voulue par l'auteur. Dans ce but, le lecteur doit effectuer une saisie intelligente de ce que l'auteur veut communiquer, de ce qu'il affiche par et dans son texte. Il doit appliquer une stratégie qui lui permet de dégager le sens, tel que l'auteur le veut, en tirant partie de la mise en texte (énoncé), mais aussi de la mise en page (présentation formelle), ce qui l'oblige à avoir une interprétation fondamentalement contextuelle, voire historique (même si l'histoire est récente). Pour bien assurer sa démarche, il doit souvent connaître l'auteur, ses idées, son appartenance (école de pensée), le contexte de production. C'est l'approche historiciste, celle qui ramène tout texte à son contexte originel.

À ce dogme (littéraire et communicationnel) de l'intention supposée de l'auteur s'oppose la thèse de l'«intentional fallacy» (ou «illusion intentionnelle») selon laquelle cette invocation est une imposture. On peut lire un texte sans se préoccuper de ce que l'auteur veut dire. Le sens n'est pas signification. L'intention n'est pas préméditation, pour reprendre les mots d'Antoine Compagnon ${ }^{8}$. C'est, en littérature, la thèse de la «mort de l'auteur»; en documentation, simplement lévidence de son absence. Cela entraîne le retour, pour certains, du texte lui-même (objet réel à observer), pour d'autres, du lecteur non pas

COMPAGNON, Antoine - $i d$ - p. 54

8. Cf. les titres de chapitres de son livre Le Démon de la théorie, op. cit.
«Avec le discours écrit, l'intention de l'auteur et l'intention du texte cessent de coïncider [... Dès lors, ] ce que dit le texte importe davantage que ce que l'auteur a voulu dire »

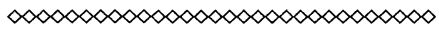

unique, mais singulier (d'où la polysémie possible de la lecture).

\subsection{Le sens est physiquement - linguistiquement - inscrit dans le texte}

Quand un auteur veut communiquer un savoir, il doit l'écrire - le coucher - sur un support. Il doit créer une trace, celle qui reste après lui. Cela est possible par le recours à l'écriture qui fige le texte. C'est la raison même de l'écriture par l'auteur. «Avec le discours écrit, l'intention de l'auteur et l'intention $d u$ texte cessent de coïncider [... Dès lors,] ce que dit le texte importe davantage que ce que l'auteur a voulu dire», écrit Paul Ricœur9.

Cette approche ne fait aucune part au cadre de la production du texte, à savoir l'«intention» de l'auteur et la «temporalité». Elle ignore ce que l'auteur veut dire - signifier - derrière les mots. Elle regarde le «dire». Elle reste à la surface. Elle écarte de même tout élément lié à la réception. Elle considère que le lecteur n'attend rien, n'a aucun projet. C'est un être abstrait, parfait, sorte de pâte qui ne demande qu'à être façonnée, qui se laisse tout simplement imprégner par ce contenu en le lisant, et doit se conformer à ce que le texte raconte. L'important n'est certainement pas l'information qu'il reçoit en tant que lecteur. C'est le contenu du texte. Cette décontextualisation fait du texte "un monument verbal au statut ontologique aussi séparé de sa production que de sa réception", selon Antoine Compagnon ${ }^{10}$.

Cette approche aborde le contenu comme quelque chose d'universel. Celui-ci acquiert ainsi un statut particulier, que certains traduisent par l'expression "savoir de l'humanité», ce qui, corollairement, lui confère un caractère désincarné, intemporel, scientifique. C'est ce qui, aux yeux de beaucoup, fait l'intérêt de cette approche. Dès lors, la lecture doit être totalement mobilisée par la recherche de ce contenu: Le texte parle de quoi? Que traite-t-il? Quel est son sujet?

Cette approche s'en tient au texte, uniquement au texte et à son aspect matériel. Celui-ci devient un objet
9. Cité In. COMPAGNON, Antoine - id - p. 96.

10. COMPAGNON, Antoine - id - p. 165 . 
extérieur aux acteurs, détaché de tout contexte, et porteur de signes dont le sens est permanent, inhérent et immanent. Le contenu inscrit dans le texte s'impose $\mathrm{du}$ seul fait qu'il est "extérieur». C'est justement ce que son lecteur doit trouver. En paraphrasant ce qu'écrivait Gaston Bachelard sur le «réel », lire ce n'est pas ce que l'on pourrait lire, mais ce que l'on aurait dû lire.

C'est sur ces trois constats que les tenants de l'analyse et de l'indexation automatiques, généralement des informaticiens, fondent leurs travaux et conçoivent des automates (les moteurs de recherche, par exemple).

Cette approche "positiviste», «objective» - on pourrait aussi bien dire «documentaire» - ne laisse plus, dès lors, aucune liberté au lecteur. Et cela est indiscutable pour beaucoup, surtout si le texte est scientifique. Mais, d'une part, cela soulève le problème de la «scientificité» d'un texte, donc des critères qui la fondent, et parallèlement celui d'une typologie des textes. D'autre part, cela laisse totalement ouvert le problème de la lecture des textes non scientifiques. Peut-on admettre cette distinction? Y a-t-il une lecture «scientifique» (objective) et une lecture «non scientifique» (littéraire ou subjective) des textes? Enfin, et surtout, cette lecture «objective» est-elle possible? Une telle idée est vivement contestée par des chercheurs aussi différents que les Allemands Wolfgang Iser et Hans Robert Jauss, l'Américain Stanley Fish, l'Italien Umberto Eco, ou encore le Français Roland Barthes. Tous, à leur façon, dénoncent ce que cette approche recèle comme illusions, illusion de l'objectivité de la lecture, illusion de l'intemporalité de son contenu (doctrine essentialiste) et illusion de l'autonomie du texte. Ils donnent au contraire toute liberté au lecteur. Ils font l'apologie du lecteur.

\subsection{Le sens est construit par le lecteur}

Lire est une activité stratégique de compréhension, donc de recherche de sens. Lire, c'est faire du sens, c'est rendre intelligible, c'est intégrer, c'est comprendre ce qu'on lit par relation. C'est donc dire que lire est une activité proprement humaine. Seul l'homme lit! et seul l'homme recherche (ou crée) du sens.

\subsubsection{Le lecteur est acteur}

Dès lors, le sens est toujours sens pour quelqu'un, pour un lecteur qui a une capacité d'intelligence, celle qui lui permet de construire une représentation occurrente (sans doute par attribution plutôt que par extraction du texte) et cohérente avec sa propre expérience, donc de comprendre et d'intégrer ce qu'il reçoit. La recherche de sens appartient toujours à celui qui lit. Faire sens est alors fonction de la compé- tence du lecteur, fonction de ce qu'il est, fonction de ce qu'il sait déjà, fonction de l'organisation de son savoir. Chaque lecteur réalise sa propre "production du sens ", selon l'expression de Roland Barthes. La lecture est singulière.

Par ailleurs, tout lecteur inscrit son activité de lecture dans un cadre, un moment, une histoire. Il n'y a jamais désert d'information, jamais nouveauté absolue. Au contraire, la lecture est motivée par un certain nombre d'attentes liées à l'expérience, ce que Hans-Robert Jauss appelle «l'horizon d'attente du lecteur ${ }^{11} »$.

Ainsi, tout lecteur consulte un texte documentaire sur la base d'une attente informationnelle, d'un besoin informationnel. Autrement dit, ce qui fait sens pour lui est fonction de l'intégration qu'il réalise des informations reçues avec ce qu'il possède déjà. C'est ce qui fait dire à certains chercheurs que toute lecture repose sur un projet. Pour comprendre un texte, tout lecteur doit avoir un projet sur ce texte, car, selon Antoine Compagnon, la compréhension d'un texte suppose l'anticipation de sens (la précompréhension). Et, selon Martin Heiddeger, cette précompréhension est inséparable de l'existence de chacun, de son être; elle est étroitement dépendante de la situation historique. Il ajoute: «le sens est ce sur quoi ouvre la projection structurée par les préalables d'acquis, de visée et de saisie et en fonction de quoi quelque chose est susceptible d'être entendue comme quelque chose ${ }^{22}$ ". De l'empathie, on est passé au projet, voire au préjugé, car souvent «la réponse que le texte apporte dépend de la question que nous lui posons de notre point de vue historique, mais aussi de notre faculté de reconstruire la question à laquelle le texte répond ${ }^{13} . .$. »

Cette hypothèse du projet oblige à considérer l'intention ou la finalité du lecteur. La lecture devient téléologique («telos»: but). Elle doit répondre au projet de celui qui lit. La recherche de sens vise alors ce qui est attendu par le lecteur, soit de façon aléatoire («je prends ce que je trouve»), soit de façon ciblée ( «je trouve ce que je cherche»). La réussite de la recherche peut être appréciée par l'accroissement du savoir antérieur ou la modification de l'attitude, selon la thèse discutable du behaviorisme. Concrètement, lire consiste à tester une hypothèse (ce que Umberto Eco appelle le «topic») en la confrontant avec le texte, à vérifier le résultat en simplifiant, en anticipant ce qui est attendu par rapport à ce qui est lu.

Dans cette approche, le lecteur se substitue à l'auteur. Il devient acteur, indépendant, totalement libre. Il retient tout ce qui fait sens pour lui à partir de son projet sur le texte. Sa lecture est ouverte: il peut choisir ce qui l'intéresse à partir de l'empreinte

\footnotetext{
11. JAUSS, Hans Robert Pour une esthétique de la réception - Paris: Gallimard, 1978.

12. cité In. COMPAGNON, Antoine- $i d$ - p. 70 .

13. cité In. COMPAGNON, Antoine- id - p.72.
} 


\section{Le contexte de production et le contexte de réception d'un texte}

Un lecteur peut-il comprendre un texte qui lui est étranger historiquement et culturellement? Peut-il comprendre un texte hors de son contexte de production? Autrement dit, un texte signifie-t-il la même chose au moment où il est produit et au moment où il est lu? (cf. la contestation sur l'identité de sens par Platon dans Phèdre.) La distance temporelle n'enlève-t-elle pas tout le sens au propos? Un texte signifie-t-il la même chose quand il est sorti de son cadre culturel de création? Autrement dit, les référents (codes, valeurs) sont-ils aussi universels que cela? Si non, le «village planétaire» n'existe plus!

Or l'idée d'«empathie » interdit toute compréhension d'une œuvre en dehors du contexte de production. Ou oblige à reconstruire le contexte (ce qui est le but de la philologie). Mais est-ce possible? Non, selon H.-G. Gadamer du fait de l'historicité de l'homme. Celui-ci serait condamné à seulement interpréter ${ }^{1}$.

Le débat est ici entre compréhension et interprétation: "ce qui déclenche l'interprétation ce n'est pas la question "Que dit le locuteur?" mais la question "Pourquoi le locuteur, dans le contexte actuel, dit-il ce qu'il dit?" L'interprétation procède d'une interrogation portant non pas sur l'énoncé, mais sur l'énonciation ${ }^{2}$.» On retrouve la distinction entre "analyse du contenu» et "analyse de contenu», entre une lecture plus littérale et une lecture nettement inférentielle. On sait, depuis Jean Meyriat ${ }^{3}$, que les documentalistes défendent l'analyse du contenu au nom de la fidélité au texte. Ils laissent l'analyse de contenu aux gens de communication.

Un lecteur peut-il, parallèlement, échapper au contexte de la réception, c'est-à-dire à lui-même et à son environnement? Non, si l'on considère que la lecture est une pratique culturelle, au sens où il n'y a pas de lecture naïve, innocente, transparente, pré-culturelle, hors de toutes références extérieures à elle, décontextualisée ${ }^{4}$. La lecture est intrinsèquement le lieu de rencontre de deux cultures. L'une est celle de celui qui conçoit le texte. L'autre est celle de celui qui le reçoit. Chacun des acteurs arrive avec ses propres normes et valeurs. Cette rencontre ne va donc pas de soi. Sauf pour ceux qui ne considèrent que le texte et son contenu.

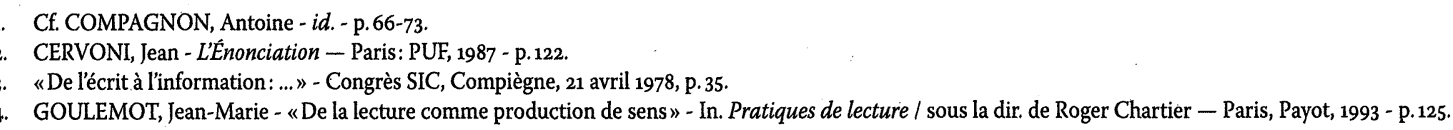

réalisée sur lui (attitude auto-plastique), ou choisir ce qui, à ses yeux, paraît important et susceptible d'agir sur l'autre (attitude allo-plastique), ou encore ce qui, à son avis, paraît être le contenu informationnel spécifique - explicite et implicite - à communiquer car susceptible d'être utile à l'autre (attitude documentaire). Dans tous les cas, la lecture sera qualifiée de «subjective».

\subsubsection{La lecture est subjective}

Lire est toujours une activité singulière. «L'idiosyncratie du lecteur - ce que nous appellerons, selon le cas, son histoire, ses intérêts, ses compétences - entre en conflit avec la structure textuelle du message et démultiplie les perspectives", écrit Jacques Leenhardt $^{14}$. "Le sens est donc un effet dont le lecteur fait l'expérience, et non un objet défini, préexistant à la lecture [...] en d'autres termes le texte instruit et le lecteur construit ${ }^{15}$.» Cela remet en cause la place de l'objet-texte et la prééminence de l'auteur.

En littérature, c'est la thèse défendue par Wolfgang Iser pour qui il y a conflit entre «conscience raisonnante» et «libre jeu de l'imagination » autour du texte. Il cherche, pour résoudre le conflit, une «structuration médiatrice». En communication, cette logique

14. LEENHARDT, Jacques La Lecture littéraire - Paris: Clancier-Guénaud, 1988.

15. COMPAGNON, Antoine - id - p. 176. interprétative du lecteur est défendue, entre autres, par Erving Goffman et Paul Watzlawick.

Ainsi, attribuer au lecteur un rôle essentiel dans la construction de sens conduit à considérer le sens que chaque lecteur se construit comme légitime. Comme il n'y a plus une seule vérité scientifique, il n'y a plus une seule lecture. Il y a seulement des interprétations, des projections. La subjectivité devient un facteur inhérent à toute recherche de sens, et donc à toute lecture faite par l'homme. Les expériences sur la cohérence - externe et interne - en lecture documentaire montrent l'importance de ce phénomène ${ }^{16}$. En effet l'homme, quoi qu'il fasse, n'est jamais neutre. On ne voit pas d'ailleurs comment il pourrait en être autrement, et comment les documentalistes pourraient échapper à ce constat.

Mais cela ne justifie pas pour autant qu'il puisse faire n'importe quoi et le déclarer «vrai». Sinon c'est l'individualisme sectaire, c'est l'enfermement intellectuel, c'est la vérité individuelle. Or de telles attitudes seraient inacceptables socialement et intellectuellement. Jean-Michel Besnier exprime parfaitement ce point de vue dans sa critique de la sensation comme moyen pour l'homme de comprendre. Celle-ci:

16. Voir les nombreuses expériences sur cette «consistency» réalisées par les psychologues et les «information scientists» américains dans les années 1950 et 1960, dont la revue American Documentation, principalement, s'est fait l'écho durant toute cette période. 
Sur le plan théorique, la liberté de choix dans la lecture pour un documentaliste dépend étroitement du schéma théorique qu'il prend comme référent implicite ou explicite de son activité.

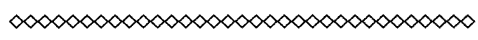

«1) est toujours vraie pour celui qui l'éprouve; autrement dit, elle rend l'erreur impensable; 2) si la science se réduit à la sensation, il n'y a pas d'accord possible entre les hommes; rien n'existe en soi qui permettrait un tel accord, et chacun est le jouet de ses sens, lesquels modifient même à tout moment l'identité du sujet qui prétend se confier à eux pour connaître [...] confondre la science avec la sensation, c'est s'interdire la vérité et l'erreur, l'objectivité et l'intersubjectivité, le langage et l'effort conceptuel - c'est-à-dire les éléments minimaux que paraît requérir l'ambition de connaître ${ }^{17}$."

Aussi la prééminence donnée au lecteur est-elle contestée. Contestée par ceux pour qui le lecteur est comme une matrice qui reçoit une impression du texte. Sans être entièrement passif, il est conduit là où le mène le texte, et donc l'auteur. Contestée aussi selon l'idée (formalisme) que tout texte est «autosuffisant», clos et stable, indépendamment de toute lecture. Contestée et combattue (positivisme), enfin et entre autres, par les documentalistes car, alors, il n'y a plus aucun moyen d'établir la validité (ni l'invalidité) de la lecture d'un texte. C'est l'illusion affective! C'est la polysémie consacrée! C'est la ruine de l'analyse documentaire!

Quel est le sens à rechercher?

Certes, tout le monde s'accorde pour dire que lire ne veut pas dire créer n'importe quel sens ${ }^{18}$. Que cela ne permet donc pas de faire n'importe quoi, sinon on passerait de «l'acte connaissant» à "l'acte délirant», selon la formule de Jean-Louis Le Moigne ${ }^{19}$. En revanche, personne ne dit celui qui est attendu parmi les sens possibles. Or trois sont possibles: le sens voulu par l'auteur, vu comme le diffuseur intentionnel d'un savoir à transmettre; le sens inscrit dans le texte, lequel s'impose comme tel car tout le monde peut le consulter; ou le sens perçu ou construit par le lecteur «effectif» qui se voit ainsi attribuer exclusivement toute capacité de production de sens.

Leur défense et/ou leur contestation traduisent une priorité implicite ou explicite accordée à l'un de

17. BESNIER, Jean-Michel Les Théories de la connaissance - Paris: FlammarionDominos, 1996 - p. 22.

18. GIASSON, Jocelyne - La Lecture: de la théorie à la pratique - Boucherville: Gaëtan Morin, 1995 - p. 13.

19. LE MOIGNE, Jean-Louis Les Épistémologies constructivistes - Paris: PUF, 1995 - p. 67 (Que sais-je? 2969). ces éléments et à la façon de le considérer: intention de l'auteur vs illusion intentionnelle; texte autonome, intemporel, indiscutable, donc "autosuffisant» vs illusion référentielle de l'objet-texte; lecteur-acteur $v s$ lecteur passif. Elles traduisent aussi la part de liberté que l'on accorde à celui qui recherche le sens par la lecture, le lecteur, selon qu'il privilégie l'auteur du texte ou l'objet-texte et son contenu (approche «objective»), ou le producteur de sens, le lecteur lui-même (approche «subjective»). Mais y a-t-il vraiment une possibilité de choix dans le processus de création de sens par la lecture? Pour ceux qui clament l'inhérente subjectivité, elle est entière. Pour ceux qui, comme les documentalistes, prônent la totale objectivité, elle est, au contraire, nulle. Cette position, évidente à priori, est-elle indiscutable?

\section{La liberté de choix dans la recherche de "sens » du documentaliste}

Sur le plan théorique, la liberté de choix dans la lecture pour un documentaliste dépend étroitement du schéma théorique qu'il prend comme référent implicite ou explicite de son activité. Or deux schémas sont possibles. L'un, le schéma «classique», l'oblige à être neutre puisqu'il n'est que "passeur» ou «intermédiaire» entre l'auteur et le lecteur final. L'autre, de vision plus interactive, conduit à contester cette neutralité. De ce fait, sa médiation, néanmoins indispensable, ne sera légitime aux yeux du lecteur final que si elle est négociée.

\subsection{La lecture documentaire selon le schéma "classique » ou la liberté « obligée »}

\subsubsection{Le schéma «classique»}

Selon la «théorie de la communication», appelée aussi «théorie du vecteur» par Robert Escarpit ${ }^{20}$, le processus de communication est généralement découpé en une série d'actes élémentaires et successifs que l'on peut analyser séparément.

Un message - séquence aléatoire, mais signifiante, de signes - est transmis d'une entité émettrice vers une entité réceptrice. Plus précisément, un émetteur commence par retrouver, dans sa mémoire, des «signifiés» («objets» ou «événements»). Il puise ensuite dans son répertoire de signes - ou codes - un nombre « $n$ " de ceux-ci permettant de les représenter. Enfin, il les assemble en une séquence appelée «message». La transmission de ce message se fait par

20. ESCARPIT, Robert Théorie générale de la communication. Paris: - Hachette Univ., 1976 - p. 23. 


\section{Tableau 2. Lecture et réécriture informationnelles du texte. Schéma « classique »}

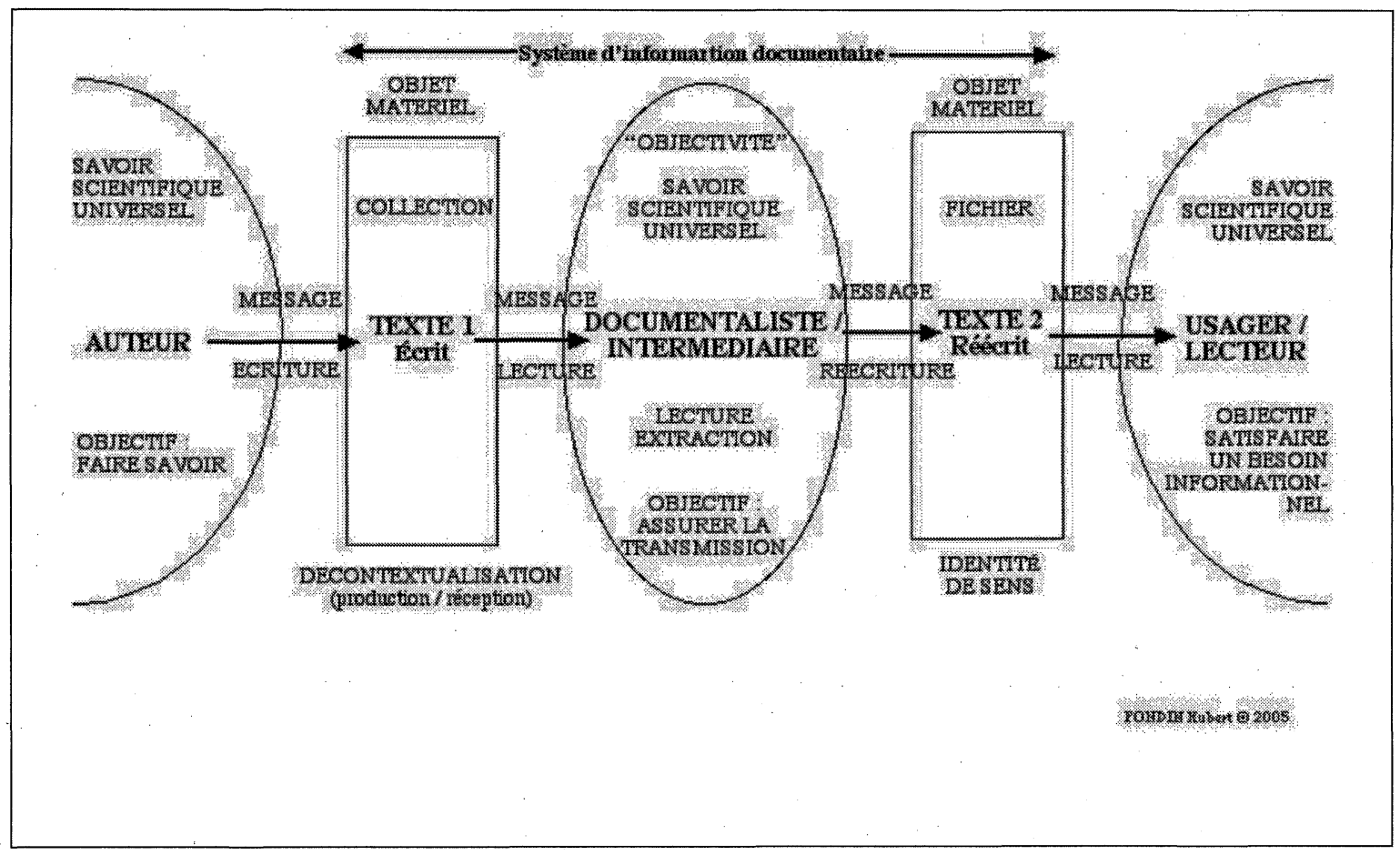

modification de son état initial - grâce à un apport d'énergie, physique, électrique ou autre - , par l'intermédiaire d'un canal.

À l'autre bout, le récepteur effectue la reconnaissance du contenu du message, par correspondance des signes - codes - avec ceux qu'il possède dans son propre répertoire, puis identifie des «signifiés» par confrontation avec sa mémoire de «signifiés» («objets» ou «événements»). Il y a alors pour lui soit nouveauté, soit redondance.

Implicitement donc, la source et la cible sont des hommes capables de prévoir et de raisonner, et non des machines. C'est la distinction forte que Robert Escarpit propose entre "processus énergétique» et «processus informationnel» (id., p. 26) ${ }^{21}$.

La représentation schématique de ce processus - tout au moins celle proposée par Claude E. Shannon - prend la forme d'un graphique linéaire, une chaîne vectorisée, dans laquelle l'accent est mis sur la transmission du message, et sur les moments de cette transmission.

\subsubsection{Les implications du schéma «classique»}

Sur la base de ce schéma, on tire trois enseignements qui, en pleine cohérence, fondent toute l'activité des spécialistes du traitement de l'information.

21. Pour Claude E. Shannon, le destinataire peut être aussi bien une personne qu'une machine (cf. sa "théorie mathématique de l'information»).

\subsubsection{Le message est porteur d'une "connaissance communiquée», l'information}

L'homme, du fait de son intelligence, acquiert par contact avec le monde environnant des connaissances - «signifiés organisés» - que, par nature, il cherche à communiquer à autrui. Celles-ci deviennent, pour l'autre, des «informations». L'information est ainsi une «connaissance communiquée» dans un message par un émetteur humain et reçue au cours d'un processus de communication par un observateur, lui aussi humain ${ }^{22}$. Jean Meyriat la définit comme «le contenu cognitif du processus de communication ${ }^{23}$ ", lequel cohabite avec un contenu affectif, volitif, ou émotif.

\subsubsection{La communication est intentionnelle}

Si l'auteur produit un message, c'est naturellement pour dire, pour communiquer quelque chose à quelqu'un d'autre. Il participe ainsi à la construction et à la diffusion du savoir. Il apporte sa contribution. Il exprime de la sorte une intention, celle de faire savoir. La communication ne comporte aucun autre enjeu qu'informationnel (les autres enjeux sont évacués).

22. Le Grand Larousse Encyclopédique (tome 11, 1970) retient cette acception quand il définit l'information comme: «nouvelle, renseignement que l'on communique ".

23. MEYRIAT, Jean - Information $v s$ communication? - In. L'Espace social de la communication - Paris: CNRS Éditions, 1985 - p. 65. 


\subsubsection{L'intention est reconnue par le récepteur-lecteur}

Dans la lecture informative d'un texte, le lecteur, documentaliste ou lecteur final, accepte un certain nombre de postulats : l'auteur veut faire savoir quelque chose; cette intention est couchée dans un texte, celui justement qu'il veut lire. Cette intention est d'autant mieux reconnue que le message est qualifié de «scientifique» ou «technique».

Les acteurs et leurs fonctions sont bien identifiés. Le message est porteur de quelque chose, un contenu cognitif, une information, une "connaissance communiquée», raison même de la communication. L'intention de communication est évidente et reconnue par le récepteur.

Certes pour le documentaliste, le problème n'est pas de savoir si le message parvient bien au bout de la chaîne tel qu'il est émis au départ. Il n'est pas d'ordre technique. Pour lui, le problème est essentiellement celui du sens, de rechercher le sens, de trouver le sens du texte qu'il lit. C'est la raison même de sa lecture, puisque cela lui permet ensuite d'en proposer des réécritures diverses (mots-clés ou résumés). Et cette préoccupation est d'autant plus forte qu'il agit en intermédiaire, qu'il agit comme un passeur. Il n'a pas à juger, à évaluer le contenu. Il doit juste permettre aux autres d'y avoir accès. Ce rôle oblige tout documentaliste à lire un texte de façon «objective». D'où l'importance qu'il donne à l'objet, à la trace, au sens «inscrit». C'est implicitement le discours qui est véhiculé dans les formations professionnelles à l'analyse documentaire, c'est aussi celui de nombre de membres de jurys de concours comportant une telle épreuve. Mais le discours correspond-il à la réalité ? Autrement dit, la lecture objective est-elle vraiment possible?

\subsubsection{La lecture objective est-elle possible?}

Cette idée d'«objectivité » repose sur le principe que tout objet (ce qui est placé devant), du fait même qu'il est extérieur à un observateur humain peut - doit - être regardé de manière neutre, impartiale, puisque c'est le même objet pour tous. On parle de "vérité» scientifique. Autrement dit, tout lecteur - tout documentaliste ou tout lecteur final - doit avoir la même saisie d'un texte, puisque que c'est le même texte pour tous. Tout lecteur doit toujours lire la même chose qu'un autre s'il a le même projet. Cela aboutit à une sorte de mise entre parenthèses du lecteur en tant que personne. Celui-ci doit se faire oublier dans sa singularité. Il doit simplement veiller à rechercher une "vérité» universelle, celle du savoir, celle de la connaissance communiquée dans le texte à lire.

C'est, pour certains, un objectif crucial pour la documentation car cela permet de réduire, d'éli- miner même le facteur humain. En effet, l'homme est inconstant, voire incohérent dans sa lecture, toutes les études de cohérence («consistency») le montrent. Dès lors, étant donné le rôle du documentaliste, pour que sa lecture soit légitime, il faut qu'elle soit conforme à une image d'invariabilité, forme de dépersonnalisation.

Mais cette posture ne fait pas - plus - l'unanimité car cela paraît un objectif difficile, sinon totalement impossible à atteindre. Pour beaucoup, il relève de l'«utopie scientifique», celle qui pense pouvoir mettre l'homme entre parenthèses. Cette posture, qualifiée d'historiciste ou de positiviste, reflète ainsi une double illusion:

$\triangleright$ celle que tout individu - ici tout lecteur élimine ses préjugés quand il veut chercher, lire ou comprendre quelque chose; celle qu'il peut regarder quelque chose tout en restant en dehors; qu'il est dès lors neutre, donc transparent;

$\triangleright$ celle que le texte existe hors du lecteur; qu'il est en soi un objet identifiable dans sa forme et son contenu, et que cela lui confère un caractère universel, autrement dit que tout lecteur, devant le même objet, doit retrouver les mêmes éléments.

Elle est combattue par la posture existentielle selon laquelle toute compréhension s'effectue sous la poussée de la motivation, sous l'influence de la préconception, "sous une visée préalable du $\operatorname{sens}^{24}$ »; par la posture constructiviste pour laquelle l'objectivité - vérité absolue - n'existe pas quand l'homme est acteur, car l'observateur appartient à l'observation, ou le lecteur à la lecture, et quand la recherche est une recherche de «sens»; et par la posture relativiste selon laquelle le lecteur ne possède jamais tous les éléments de connaissance sur l'objet analysé. Il en prend connaissance avec ce qu'il est et ce qu'il sait. Il est donc amené à choisir, à privilégier, délibérément ou non. C'est donc qu'une recherche de «sens», qu' «une compréhension est toujours de l'ordre $d u$ possible et $d u$ "peut-être". Elle peut être correcte ou juste, mais jamais vraie ou fausse», selon Marc-A. Ouaknin ${ }^{25}$.

Ainsi il est difficile d'imaginer qu'il existe une seule lecture documentaire d'un texte, une lecture vraie, autrement dit unique, celle qui s'impose à tout lecteur. Et il est très difficile, voire impossible, d'afficher le modèle "standard" d'une telle lecture, même si nombre d'étudiants et de correcteurs d'épreuves en analyse documentaire en demandent un. Ce constat, si on le suit, ne conduit pas à une contestation systématique de la production documentaire. Mais il oblige à s'interroger sur la vision de la lecture documentaire chez les documentalistes, et donc à reconsidérer le cadre théorique "classique» qui en constitue le socle. Car toute pratique doit pouvoir être interrogée sauf

24. GADAMER, Hans- Georg - L'Art de comprendre - Paris: Aubier, 1982 - p. 82. 25. OUAKNIN, Marc-A. La Bibliothérapie - Paris: Seuil, 1994 - p. 246. 


\section{Tableau 3. Lecture et réécriture informationnelles du texte. Schéma « interactif »}

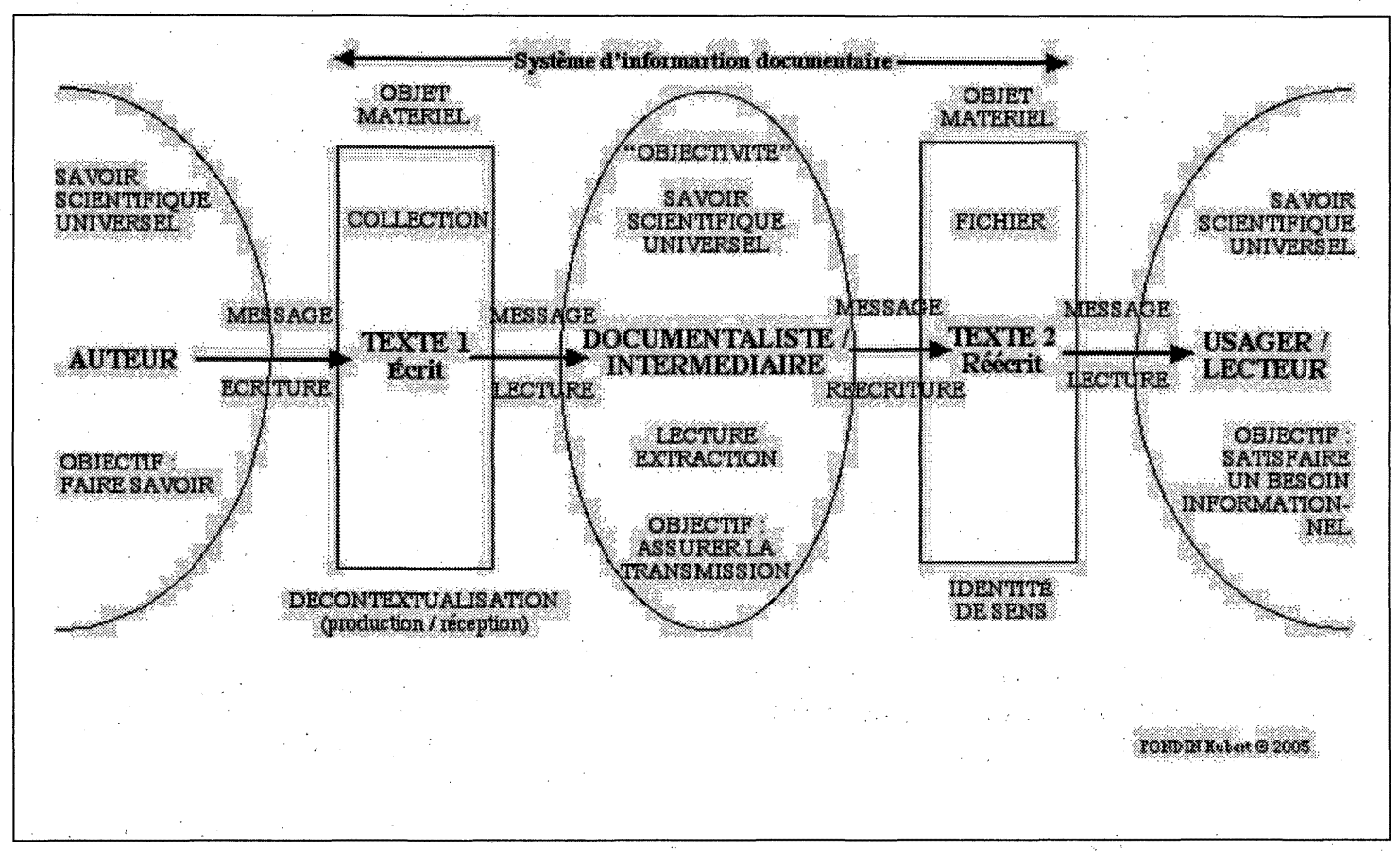

à considérer que la documentation est décidément un monde à part avec ses propres règles et ses fortes certitudes.

Pour voir autrement, il faut sans doute changer de cadre théorique.

\subsection{La lecture documentaire selon le schéma interactif ou la liberté partagée}

Personne ne vit dans une bulle, sans contact avec l'autre. L'homme est un être social qui a besoin de communiquer, de partager. Entre les hommes, il y a nécessairement une relation, un échange, une expression de la vie. Il y a un phénomène communicationnel. Il y a nécessairement place pour quelque chose de commun, y compris dans la lecture.

Ce qui est en cause, ce n'est donc pas en soi cette pratique, car cela fait longtemps que sa place est reconnue. Ce qui parait en cause, c'est le cadre théorique dans lequel on l'enferme. On a recours à une approche analytique. On s'appuie sur une vision linéaire. On focalise l'attention sur le message, le texte, son contenu. Ce faisant, on semble oublier que la "signification", c'est aussi un cadre, un contexte, et des acteurs avec leurs enjeux. Or cela qualifie naturellement toute activité de lecture. Celle-ci doit être vue comme un échange, un partage dans certain cas d'expériences (lecture «littéraire»), dans d'autres cas - et c'est ce qui nous intéresse ici -, de savoir (lecture «informative»). Mais c'est toujours un partage entre des hommes, à travers le temps et l'espace, entre des êtres singuliers avec leur représentation du monde, avec leurs moyens d'expression, qui, malgré tout, ont le souci de communiquer, le souci de rechercher une coexistence constructive.

Cette idée de partage prend un poids particulier dans la fonction d'intermédiaire remplie par le documentaliste. Celui-ci devient médiateur avec un double souci de partage, puisqu'il doit faire le lien entre l'auteur, d'une part, et le lecteur final, d'autre part. Il est donc au centre d'un réseau d'interactions, visibles ou invisibles, dont il ne peut pas ne pas tenir compte dans sa lecture de médiation.

La notion qui voudrait traduire cela est celle d'« intersubjectivité» qu'il convient de replacer dans le schéma interactif de la communication.

\subsubsection{Le schéma interactif}

La représentation du phénomène de communication donnée par le schéma "classique» est désormais dénoncée partout comme erronée ${ }^{26}$. La théorie qui la fonde, celle de la communicationvecteur, correspond à un besoin de recherche de la "vérité», à un besoin d'explication scientifique de la transmission d'un message quelconque. Ce n'est pas un de ses moindres mérites. Mais le modèle proposé est trompeur car trop simpliste. Il donne une fausse idée de la communication en tant que phénomène humain. Ce n'est pas une simple transmission. La

\footnotetext{
26." "Ce schéma origine-fin [est] dans la dynamique de transfert et de transposition de modèles de scientificité propres aux sciences exactes", selon Armand et Michèle Mattelart (cf. Histoires des théories de la communication - Paris, La Découverte, 2002 - p. 31-33)
} 


\section{Dans le cadre d'une communication interactive, la lecture documentaire d'un texte devient un phénomène communica- tionnel spécifique avec des caractéristiques fortes.}

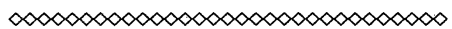

vision linéaire qu'il suggère est largement insuffisante pour en comprendre toute la complexité, toutes les logiques sous-tendues, surtout quand elles sont humaines.

\subsubsection{Les implications du schéma «interactif»}

Sur la base de ce nouveau schéma, on tire trois enseignements: la communication est un phénomène humain et global dans lequel apparaissent des jeux d'acteurs (interactions), des conditions de partage (contexte), et des enjeux d'échange (but).

\subsubsection{Une communication interactive}

La communication en tant que phénomène humain et global est irréductible à une simple transmission de signes d'un émetteur vers un récepteur (mono-directionnelle), irréductible à une répartition de rôles dans laquelle l'émetteur serait actif, et le récepteur, passif. Au contraire, celui-ci réagit au. message de l'émetteur. Chacun exerce successivement sur l'autre une influence. Il y a un phénomène de réciprocité. Une réponse surgit à partir de ce que chacun des partenaires fait, dit, pense ou lit. Se construit ainsi une interaction. Celle-ci est encore plus réelle et complexe quand le nombre d'acteurs augmente, ce qui est - virtuellement - le cas au cours de la lecture documentaire, car virtuellement il y a au moins l'auteur, le documentaliste et le lecteur final autour d'un même texte, et que - virtuellement - nombre de documentalistes dialoguent sur le même texte même si c'est à des moments différents.

\subsubsection{Une communication contextualisée}

Pour que la communication puisse s'établir réellement, les acteurs humains doivent non seulement pouvoir échanger techniquement, mais aussi et surtout partager socialement et culturellement. Pour se comprendre en "empathie», ils doivent non seulement posséder les mêmes répertoires de signifiés et de signes, être dans le même environnement, mais encore avoir les mêmes «valeurs», être «sur la même longueur d'onde». Voire être dans la même sphère, ce qui signifie le même espace et le même temps. Sinon comment peut-il y avoir réellement échange ou partage de sens?

\subsubsection{Une communication à enjeux}

La complexité du phénomène de communication tient encore à la prise en considération des facteurs humains, lesquels obéissent à des logiques diverses et traduisent de nombreux enjeux ou en sont le résultat. Vue comme un phénomène communicationnel, la lecture implique souvent "une stratégie de l'affrontement et de la manipulation ${ }^{27}$ ».

Alex Muchielli28 identifie cinq types d'enjeux communicationnels:

$\triangleright$ l'enjeu informatif (c'est normalement la première fonction de la communication, c'est la fonction référentielle de Roman Jakobson);

$\triangleright$ l'enjeu de positionnement, pour définir son identité par rapport à autrui;

$\triangleright$ l'enjeu de mobilisation visant à influencer autrui (l'argumentation);

$\triangleright$ l'enjeu relationnel (la séduction);

$\triangleright$ l'enjeu normatif centré sur la régulation des relations elles-mêmes.

Dans l'activité documentaire, l'enjeu informatif est naturellement valorisé. Mais peut-on pour autant totalement évacuer les autres enjeux (par exemple, l'édition scientifique avec les problèmes de positionnement et de mobilisation des auteurs)?

Dans le cadre d'une communication interactive, la lecture documentaire d'un texte devient un phénomène communicationnel spécifique avec des caractéristiques fortes. En effet, c'est une communication interactive dans laquelle un des acteurs est généralement absent (l'auteur), dans laquelle le contexte de production est lui aussi souvent absent, puisque la trace écrite (le document) permet de franchir les obstacles de la distance et du temps, dans laquelle, enfin, l'intention de l'auteur de faire savoir est totalement reconnue par le lecteur. C'est en outre, et c'est essentiel pour comprendre le débat, une lecture pour autrui. C'est sa finalité propre, et cela est parfaitement mis en avant par les documentalistes, même si la réalité donne parfois l'impression de ne pas cadrer avec le discours. Comment alors en assurer pleinement la réussite?

Rechercher du sens c'est, dans ce nouveau cadre, s'inscrire dans un projet qui ne peut être que la résultante des trois éléments parties prenantes dans la lecture: le texte (objet matériel), le lecteur (construction de sens) et l'auteur (intention informationnelle). Cette posture implique de mettre en avant le partage informationnel, le partage de sens. La compréhension - le sens - devient un partage, une mise en commun, de ce qui est l'objet même de

27. GOULEMOT, Jean-Marie - $i d$ - - p.116

28. MUCHIELLI, Alex Psychologie de la communication - Paris: PUF, 1995. 
l'échange d'un message, grâce à la négociation, grâce à l'intersubjectivité.

\subsubsection{La lecture "partagée» ou "intersubjective»}

\subsubsection{L'intersubjectivité}

C'est Roger Bautier qui, dès 1981, écrit que «l'objectivité scientifique n'est qu'une subjectivité partagée ${ }^{29}$ ", que c'est une sorte de négociation sur la réalité. Autrement dit, l'objectivité devient une opinion ou un regard partagé entre des hommes qui sont concernés par le même projet. Elle n'existe pas en soi, elle se construit par consensus. C'est l'idée illustrée par le concept d' $\mathrm{d}^{\text {intersubjectivité }}{ }^{30}$ ».

Jean-Michel Besnier ne dit pas autre chose quand il écrit que "bien comprise, l'objectivité scientifique décrit et consacre l'intersubjectivité des hommes de science $^{31} »$. Jean-Louis Le Moigne reprend l'idée, mais préfère utiliser le terme de "projectivité» car, pour lui, toute activité humaine est associée à un projet, d'où sa nature téléologique ${ }^{32}$. On a vu plus haut que c'était le cas de la lecture (cf. «Le sens est construit par le lecteur»).

L'intersubjectivité permet ainsi de prendre en compte à la fois le caractère «subjectif» de la lecture, c'est-à-dire l'espace de débordement reconnu à chaque lecteur du fait de sa singularité, et le souci d'une saisie «objective» du contenu du texte du fait du partage communicationnel attendu. Cette (re-)conciliation est le gage d'une «bonne» lecture documentaire. L'acte de lecture correspond ainsi à un "dialogue», avec ses objectifs et ses règles, qui reconnaît que «le texte a quelque chose à dire qui lui est propre et qui résiste à la pure subjectivité du lecteur, car si le texte n'avait pas cette force, il ne serait qu'un simple miroir permettant la projection $d u$ lecteur ${ }^{33}$ ». Le sens devient global, à la fois celui que l'auteur a voulu, celui qui se trouve dans le texte, et celui que tout lecteur attend.

\subsubsection{L'information est une connaissance non pas communiquée, mais partagée}

Un texte n'existe pas sans un lecteur, qui luimême ne peut ignorer l'auteur. Loin d'être éliminé dans la démarche de lecture, le facteur humain est, au contraire, introduit sur les bases d'un consensus d'autant plus facile à obtenir que les lecteurs - intermédiaire et final - ont tous le même objectif, le même projet: comprendre ce qu'un auteur à travers

\footnotetext{
29. Lettre d'Inforcom, Déc. 1981, p.7

30. Sur cette notion, cf. BERRENDONNER A., PARRET H. - L'Interaction communicative - Berne: Lang, 1990.

31. BESNIER, Jean-Michel, id. - p. 9.

32. LE MOIGNE, Jean-Louis, $i d$. - p. 57 et 81 et 94.

33. OUAKNIN, Marc-A. - id. - p. 246
}

son texte veut signifier. C'est normalement le cas pour un texte «scientifique». Cela aboutit à une véritable négociation dont le relevé de conclusions indique ce que tout lecteur, quel que soit son rôle, doit avoir compris dans un même texte pour qu'il puisse y avoir un réel partage sur ce texte. En conséquence, l'information n'est plus une connaissance communiquée, comme on l'a déduit à partir du schéma «classique». C'est désormais une connaissance partagée. Au transfert se substitue l'empathie.

Dans le cadre d'une lecture pour autrui (documentaire), le partage du sens d'un texte est le résultat d'une négociation entre l'intention de l'auteur et le projet du lecteur final, puisque la lecture documentaire doit fournir une réponse à ce projet. Il se construit, réellement ou virtuellement, à partir des éléments nécessaires et suffisants pour que tous les lecteurs, intermédiaire et final, se reconnaissent dans le résultat de la lecture ${ }^{34}$. Tout documentaliste rétorquera justement qu'il lui est difficile de négocier avec des absents (auteur et lecteur final). Qu'il est seul devant son texte. Qu'il décide seul de sa lecture. Mais il ne doit pas oublier que la lecture documentaire, du fait de ses caractéristiques, n'est pas une simple transmission, mais un partage, virtuel certes, mais un partage d'information. Comment peut-il faire?

\subsubsection{La mise en cohérence de la représentation du texte}

Cela établi et accepté, il convient alors d'appliquer les stratégies qui permettent de dégager l'information à partager. Le problème est désormais d'ordre à la fois psycho-cognitif, culturel et social, nullement d'ordre technique ${ }^{35}$. Une des solutions pour parvenir à ce partage est la mise en cohérence de la représentation - image mentale - du texte. Pour Jean-Michel Besnier, «si les savants ne disent plus la vérité, qu'ils déploient au moins des systèmes satisfaisants pour l'esprit. La cohérence demeure la dernière politesse des savants ${ }^{36} . »$

"La cohérence n'est pas une propriété linguistique des énoncés, mais le produit d'une activité interprétative [...] le jugement de cohérence est rendu possible par la découverte d'[au moins] une orientation argumentative globale du texte ou de la séquence», écrit Jean-Michel Adam ${ }^{37}$. L'objectif de la lecture documentaire devient donc la recherche d'une représentation intellectuelle cohérente. La recherche de sens est en fait une recherche de cohérence, à titre

\footnotetext{
34. Pour une lecture pour soi, le sens du texte devient l'intention de l'auteur dégagé à travers le projet du lecteur.

35. L'aspect technique se retrouve en revanche lors de la réécriture. Mais il faut distinguer les deux moments.

36. BESNIER, Jean-Michel - Faut-il brûler Descartes - Science et Avenir - Hors série, déc. 1999, p.13.

37. ADAM Jean-Michel - Les plans d'organisation du texte - In Les Entretiens Nathan,
} Actes II, Lecture et écriture - Paris: Nathan, 1992 - p. 139. 


\section{Pour qui considère que la lecture documentaire est une activité commu- nicationnelle de type intentionnel, donc diverse et complexe, il convient de prendre un modèle plus «intéractif» intégrant tous les acteurs, car l'homme ne peut être mis entre parenthèses.}

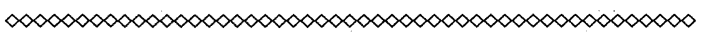

individuel et/ou à titre collectif, dans la globalité de ce qui est à lire. Cela peut se faire par la recherche d'une "rationalité procédurale, susceptible de justifier. l'accord sur les méthodes ${ }^{38}$ " avec des grilles de lecture, par exemple. On est loin d'une pratique naturelle, spontanée, évidente! C'est une pratique qui doit être encadrée, donc maîtrisée, donc apprise dans ses modalités - non techniques - de réalisation!

Il faut ainsi regarder globalement l'auteur, son projet, son intention, et sa production comme acte dans le temps; le lecteur final, son projet, sa réception et ses capacités d'appropriation culturelle et intellectuelle d'un texte «lu» par un autre; le texte, enfin, comme réalité matérielle, mais aussi comme objet linguistique, et comme élément de communication. La lecture documentaire devient le résultat de l'interaction que tout documentaliste réaliserait entre ces trois pôles dans le cadre d'une véritable activité communicationnelle de partage de savoir, avec ce qu'il est, avec ce qu'il sait... car il ne peut se prétendre neutre, transparent. Et ce biais, inévitable, sera d'autant mieux accepté par le lecteur final, que le documentaliste est pleinement conscient qu'il n'est pas un simple passeur. Il doit donc avoir le souci de fonder sa pratique. La liberté dans la lecture est désormais celle qui assure le partage communicationnel. Cette liberté devient responsabilité et, pour les documentalistes, responsabilité professionnelle.

\section{Conclusion}

Lire n'est pas une activité banale surtout quand elle est faite pour autrui, ce qui est l'essence même de la lecture informative de type documentaire, celle qui passe par le texte.

Cette activité est depuis toujours regardée à travers le cadre du schéma "classique» de la communication. Or celui-ci, entre autres, identifie l'information du lecteur, celle que doit dégager le documentaliste en tant qu'intermédiaire, à la connaissance communiquée par l'auteur de façon intentionnelle. La lecture doit donc être la plus objective possible. C'est ce que des générations de documentalistes entendent

\footnotetext{
38. BESNIER Jean-Michel, $i d$.
}

et reproduisent dans leur vie professionnelle de peur d'être accusés de «subjectivisme» ou d'«impressionnisme».

Pour autant, cette activité n'a pas acquis sa légitimité car elle est prise au piège de ce dilemme: "objectivité» versus "subjectivité» de la lecture. Ce piège, c'est justement celui dans lequel on l'enferme en prenant comme modèle le schéma «classique» de la communication.

Pour qui considère que la lecture documentaire est une activité communicationnelle de type intentionnel, donc diverse et complexe, il convient de prendre un modèle plus «interactif» intégrant tous les acteurs, car l'homme ne peut être mis entre parenthèses. Dès lors, la place et le rôle du documentaliste en tant que médiateur, dans le processus de lecture et, à travers celle-ci, d'accès à l'information, doivent être regardés à travers un nouveau cadre, celui qui met le partage comme objectif de toute communication de savoir.

Or le partage est difficile. Sa réussite n'est pas évidente, dans la lecture comme ailleurs. Comment faire pour que la lecture soit réussie? Sans doute en recherchant un consensus entre les lecteurs, une base informationnelle minimale et nécessaire. C'est ce que veut traduire le concept d' «intersubjectivité». La liberté du lecteur est donc une liberté partagée. L'information devient la connaissance partagée.

En conséquence, lire ce n'est pas seulement rechercher une adéquation entre sens voulu, sens inscrit et sens perçu. C'est surtout construire - en cohérence, en responsabilité et en toute liberté - un sens partagé, celui qui est nécessaire au maintien de la communication entre les humains et à l'échange des connaissances. Car au-delà des particularités de chaque lecteur, il y a un «sens» partageable par tous les lecteurs. Sinon il n'y a pas d'échange, pas de vie.

En concluant ainsi, nous nous sentons pleinement dans le domaine des sciences de l'information et de la communication (SIC), puisque le problème de la lecture est au croisement de considérations et d'études socioculturelles (sur les acteurs et sur le cadre), d'une part, et sémio-cognitives (sur le sens), d'autre part.

Nous nous sentons aussi pleinement dans la science de l'information (SI), puisque la préoccupation est celle de la construction d'une mémoire de nature documentaire (travail pour autrui) autour d'un véritable partage de sens (représentation et expression diverses des savoirs).

Nous pressentons enfin les changements que cela peut induire dans le contenu de nombre de formations à l'analyse documentaire, à nos yeux encore trop déterministe (même lecture et même traduction d'un même texte) et trop instrumentale (présentation et manipulation de langages documentaires). On y oublie souvent le partage de «sens». (-) 